sure to result in the indignity of having the certificate refused by the registrar." The late Mr Gavin Thurston (who preferred in his office of coroner not to be referred to by his medical title) wrote, "Cerebral haemorrhage suggests head injury, possibly homicidal, to the registrar. Further inquiry usually reveals the more usual high blood pressure." In my own experience as a coroner many so-called cerebrovascular accidents or cerebral haemorrhages have turned out after investigation to be subdural haemorrhages due to injury.

Perhaps even worse is the latest horror"chronic obstructive airways disease": in my experience this is used for anything from asthma to carcinoma of the bronchus and was recently given as a cause of death for a coalminer with a $50 \%$ industrial disability pension for pneumoconiosis. He was survived by his widow, who would obviously not benefit from an inaccurate description of the reason for his demise.

F G HaILs

Staffordshire (North) Coroner's

District,

Stoke-on-Trent ST4 6AS

1 Simpson K. Forensic medicine, 6th ed. London: Edward Arnold, 1969:208.

2 Thurston G. Coroner's practice. London: Butterworth, 1958:30.

\section{Children in wheelchairs}

SIR,-Having read Alison Wisbeach and Professor K S Holt's article "Children in wheelchairs" (19 July, p 199), I wondered if the Ortho-Kinetic chair had been considered. We find it answers many problems, especially transfer to the car. The chair becomes the car seat, with no adaptations to the car necessary and no lifting of the patients involved.

It is aesthetically pleasing and has many adaptations. Its main drawbacks are that it is only attendant operated and it is not yet supplied by the DHSS, although some local authorities are interested.

Pat FreEMaN Superintendent Physiotherapist, Lewisham Health District

Beckenham, Kent

under stress and in cases of painful metastases in bone. When designed the wheat-grain beds and cushions were intended for short-term use, but they proved very popular also in longterm use, despite their obvious disadvantages. As soon as I was able to obtain the expanded polystyrene beads to take the place of the wheat grains their lightness, warmth, and simplicity of laundering were appreciated: while the clinical effectiveness in providing a dependable and entirely comfortable medium of support eliminated the instability inherent in all forms of spring support and uncontrolled fluid media such as water and air.

Much small-team work was involved in the design and production of a generally useful and acceptable series of "granular support cushions" -neck cushions, bed cushions (from shoulders to knees), and seat cushions (for most seating problems, including driving all sorts of vehicles, and especially appreciated by the "chair-fast" for comfort and stability, preventing and healing pressure sores in difficult cases). Finally, granular support heel and calf cushions were designed at the specific request of one of the three Exeter orthopaedic surgeons, all of whom now constantly use them for fracture patients on traction in slings in practically all cases since they were introduced some three years ago.

These and the other types mentioned are now in regular use in three Exeter hospitals, by many of my own patients and those of GP colleagues, and by the welfare services of area health authorities; and they have been supplied to Cheshire homes and to the Marie Curie Memorial and other establishments.

Having developed them from personal experience of their stabilising properties for back and neck troubles, I have slept continuously on these cushions during the last 10 years and take them everywhere-they should be more widely known and used.

Pinhoe, Exeter

JOHN B TRACEY

1 Tracey JB. Practitioner 1970;204:845-6.

Tracey JB. Handbook of impact therapy. 3rd ed, 1979 supplement. Personal publication, 1979:102-10.

\section{Treatment of axillary hyperhidrosis}

\section{Seating the disabled}

SIR,-With reference to your leading article (5 April, p 963) "Seating the disabled," in my experience this is not necessarily a complicated problem, provided that basic principles of support are fulfilled-namely, maximum area of base support for best stability and balance; complete adaptation to the part supported; and a limited variability that can be produced at will to ensure comfort.

"Granular support," a term used to cover this type of supportive medium, was first used by me some 20 years ago to fulfil these criteria and so incidentally to eliminate "pressure points." In a suitably designed cushion, the friction between the granules must be sufficiently low to ensure full adaptation of the cushion to the bodily contours, in the first instance by voluntary movements of adjustment, but of a sufficiently high friction to retain the chosen position of comfort, which can be varied at will. I have described ${ }^{12}$ a welltried and simple application of these principles that gave great relief to sufferers both from seat troubles and from back pain, among many other applications in the support of joints

SIR,-I have followed with great interest the papers on treatment of axillary hyperhidrosis by Mr K T Scholes and others (8 July 1978, p 84) and by Mr C R W Rayner and others (10 May, p 1168), and the subsequent correspondence culminating in the rejoinder from $\mathrm{Mr}$ Colin Rayner to Dr W F G Tucker's letter (16 August, p 520). As my company markets a formulation of aluminium chloride hexahydrate (Anhydrol Forte) corresponding to that used by $\mathrm{Mr}$ Scholes and his colleagues I am sure your readers will be interested in the following factual observations-free, I trust, from any commercial bias.

In the first place, we absolutely confirm $\mathrm{Mr}$ Scholes's statement that it takes three weeks to prepare the $20 \%$ solution of aluminium chloride hexahydrate in alcohol. Any attempt to shorten this time results in a portion of the crystals failing to dissolve and the supernatant liquid being of a lower concentration. This has occasionally happened in hospital pharmacies, owing to the difficulty in compounding this formulation, and we have examined some samples purporting to be of full strength, but on analysis proving to have much lower levels-one as low as $12 \%$.
Much more important, however, is the fact that almost always patients will use far too much of the solution if applying it with a brush. We found in our preliminary clinical trials that severe local reactions occurred to the extent described by $\mathrm{Mr}$ Rayner and that these reactions almost completely disappeared when a roll-on applicator (which permits only a tiny calculated amount to be applied) was used instead of a brush. This is borne out by the fact that since issuing Anhydrol Forte in June no adverse reactions have been reported.

Incidentally, I cannot understand $\mathrm{Dr}$ Tucker's reference to "an aqueous solution of $20 \%$ aluminium chloride hexahydrate." It is well known that aluminium chloride hydrolyses in aqueous solution to form hydrochloric acid; that is why the injunction to make sure that the site is absolutely dry before the solution is applied must be observed if irritation is to be avoided.

Dermal Laboratories Limited,

H YARROW

\section{Aortic stenosis from calcification of congenital bicuspid valve}

SIR,-Dr N Brooks's report (9 August, p 424) on cases of rapid development of severe aortic stenosis points out the difficulties encountered by physicians in assessing the degree of stenosis clinically and sometimes even haemodynamically.

Although cases 2 and 3 showed an initial small transvalvar gradient, despite the use of the same equipment, this does not necessarily reflect insignificant stenosis haemodynamically. Heart rate and flow can be extremely variable and with a valve whose area is narrowed by around $50 \%$ may still show an insignificant pressure gradient. This results from the relationship between the flow and gradient for a particular valve area which shows a gradually diminishing slope until it becomes almost flat. Furthermore, with a very narrow valve area the curve becomes an asymptote exponential reaching a plateau at very low flows. The difference in heart rate between any two investigations carried out at different times is also very important because even though the cardiac output may be identical the systolic ejection period will be different as the rate changes.

If on clinical grounds there are indications for catheterising patients with aortic stenosis and an "insignificant" gradient is discovered, concomitant cardiac output measurement across the valve or pressure gradient measurement following a period of exercise (or both) is indicated as a small change in flow demands a large increase in pressure gradient; this relationship is more marked the narrower the valve area. Such manoeuvres may bring out a more realistic index of obstruction than resting pressure gradients alone.

J V DE GIOvanNI

Birmingham General Hospital, Birmingham

\section{The motives of fakers}

SIR,-Sir Douglas Hubble (16 August, p 523) has seriously underestimated the number of fakers exposed in the past 10 years. To his list we may add the names of Summerlin, ${ }^{1}$ Derr, ${ }^{2}$ and Gullis ${ }^{3}$ in the biological field alone. 